\title{
An old task in new clothes: A preregistered direct replication attempt of enclothed cognition effects on Stroop performance
}

\author{
Devin M. Burns ${ }^{1}$, Elizabeth L. Fox ${ }^{2}$, Michael Greenstein ${ }^{3}$, Gayla Olbright ${ }^{1}$, and DeMaris Montgomery ${ }^{4}$ \\ ${ }^{1}$ Missouri University of Science and Technology \\ ${ }^{2}$ Wright State University \\ ${ }^{3}$ Framingham State University \\ ${ }^{4}$ Bradley University \\ Affiliation
}

\begin{abstract}
Adam and Galinsky (2012) motivated their theory of enclothed cognition using experimental results showing that wearing a doctor's coat improved selective attention by reducing errors on incongruent Stroop trials. While many other studies have pursued extensions of this idea, there have been no published replications of this influence on the Stroop effect. This preregistered, direct replication attempt uses equivalence testing and the small telescopes framework from Simonsohn (2015) to argue that such an effect, if it exists, is too small to have been reliably detected under the original design. Theoretically predicted sequential effects as small as $7 \mathrm{~ms}$ were successfully detected at $\alpha=.05$, indicating that the failure to find an effect of lab coat is likely not due to power limitations or poor experimental technique.
\end{abstract}

Keywords: Enclothed Cognition, Replication, Stroop, Equivalence Testing

\section{Introduction}

A person's clothes can influence the way they feel and how other people perceive them. For instance, clothing that is in line with stereotypical expectations of a type of person or occupation (e.g., doctor, scientist) can facilitate the wearer's ability to envision themselves through the lens of the person they are impersonating: effectively "get in the role" of an altered perception of the self. This idea has been utilized by video game designers to pull a user deeper into a fictional world (Hur, Sturdee, Alonso, Markopoulos, \& Alexander, 2017, Pagliarini \& Lund, 2011). Wearing particular clothing is just one form of physical experience that can carry symbolic meaning and influence the mental representation one has of abstract concepts. There are at least two plausible pathways by which costume wear could produce subjective and behavioral changes: either through the stereotypical physical actions associated with the role-play of the depicted character, or perhaps stemming directly from the costume itself.

Embodied or grounded cognition (Barsalou, 2010) describes how engagement in a type of physical activity (e.g., cleaning, change of body posture) or perceptual experience

Correspondence concerning this article should be addressed to Devin Burns, Missouri S\&T, 500 W. 14th St., H-SS 113, Rolla, MO 65409. Email: BurnsDe@mst.edu (e.g., scents, warmth) can directly alter subsequent judgments and behaviors (Lee \& Schwarz, 2012). Grounded cognition suggests a link between the stereotypical symbolic meaning of the action and the underlying portrayal of self, others, and/or an environment (e.g., feelings of importance and power). This relationship is shown to extend beyond the perceptions of the participant to also impact how others perceive and behave toward them. For instance, students rate professionally dressed teachers as more intelligent and those who are casually dressed as more interesting (Morris, Gorham, Cohen, \& Huffman, 1996).

Adam and Galinsky (2012) investigated whether clothing can affect the wearer in the absence of any associated physical actions, asserting that "clothes can have profound and systematic psychological and behavioral consequences for their wearers." They argued that this can occur when the clothes are imbued with symbolic meaning. Specifically, their data suggest that the association between a doctor's coat and intelligence can lend the wearer an increased ability in selective and sustained attention tasks. They concluded that clothing is capable of not only influencing what we think, but also the abilities and mechanisms associated with how we think, a phenomenon they called enclothed cognition.

In their first of three experiments, Adam and Galinsky (2012) measured selective attention using the Stroop task (Stroop, 1935), which has been referred to as the gold standard of attentional measures for its robustness and broad ap- 
plicability (MacLeod, 1992). The basic design employs stimuli made of a color word that can appear in different font colors, with participants asked to indicate the color of the font while ignoring the word itself. Stroop interference occurs when participants perform worse with incongruent stimuli (e.g., the word "RED" in blue font) compared to neutral stimuli (e.g., the string "XXXX" in blue font). A related effect of Stroop facilitation occurs when participants perform better on congruent stimuli (e.g., "BLUE" in blue) than on neutral stimuli. Adam and Galinsky's data revealed that participants who wore a lab coat made fewer errors on incongruent trials than participants in the control group, indicating that wearing a lab coat can increase attentional performance.

One potential alternative explanation for these results is that merely priming the concept of "doctor" could be enough for the effect, and wearing the clothing is superfluous. In experiments 2 and 3 Adam and Galinsky sought to distinguish between these explanations by comparing the effects of merely seeing the coat to those of wearing it. They also attempted to confirm that the effect was restricted to situations where the coat had strong symbolic associations with intelligence by including a condition where participants wore the same coat but were told it was a painter's coat. Their results indicated that participant's performed better when wearing a "doctor's coat" rather than merely seeing the coat or wearing a "painter's coat."

In these two experiments they measured what they called sustained attention (rather than selective attention) using a comparative visual search task where participants were instructed to find the four differences between a pair of detailed photographic images. This paradigm was inspired by Pomplun, Reingold, and Shen (2001), though there are many important differences in methodology. This type of task has not been previously used to quantify sustained attention, making it somewhat challenging to interpret the results.

Given that the Stroop paradigm is well recognized as the standard for selective attention research, this replication attempt will focus on the results from experiment 1 and not experiments 2 and 3 . Additionally, experiments 2 and 3 were intended to resolve whether the lab coat effect could be attributed to just wearing a coat without the association to doctors or to merely seeing the coat. This replication attempt seeks to address the primary concern of whether or not there exists a measurable effect of wearing a lab coat, the answer to which may obviate the attributional question. If such an effect were to be found, however, then replicating these experiments as well may prove fruitful.

\section{Subsequent research}

Other investigators have applied the theory of enclothed cognition to a range of different domains. One of the first published articles attempting to provide additional evidence for the theory came from Van Stockum and DeCaro (2014), who investigated how wearing a lab coat may impact insight problem solving. They hypothesized that the higher level of attentional control granted by enclothed cognition would impair performance on insight problems, consistent with other research that indicated that individuals with higher Working Memory Capacity (WMC) performed worse than their peers on insight problems (DeCaro, Van Stockum, \& Wieth, 2016).

As in Adam and Galinsky's second and third experiments, half of the participants who wore coats were told that they were painter's coats instead of doctor's coats. In contrast to the original result, however, Van Stockum and DeCaro (2014) found no difference in performance depending on the label attached to the coat. They also failed to find the predicted main effect of WMC or a main effect of lab coat. The only significant effect found was an interaction between WMC and lab coat (pooled across the two different labels) such that for low WMC participants those wearing a coat scored lower on insight problems, but amongst high WMC participants the coat was associated with improved performance. These results provide meager support for the enclothed cognition hypothesis in that there was no overall effect of wearing a coat across individuals and any such effects did not depend on the significance attached to the coat, as operationalized in the doctor versus painter manipulation.

While Van Stockum and DeCaro (2014) predicted that wearing a lab coat could hinder the abstract processing required for solving insight problems, Slepian, Ferber, Gold, and Rutchick (2015) presented five experiments indicating that wearing formal clothing may increase participants' tendency to use abstract processing. Their first, second, and fifth studies showed a relation between abstract processing and formal clothing, but did not randomly assign clothing, leaving open the possibility of preexisting differences between participants who tend to wear formal or casual clothes. The third manipulated clothing formality and found a significant effect on category inclusiveness, but only had a limited sample of only 34 participants. Their fourth experiment had 54 participants and was the most comparable to the attention measures of Adam and Galinsky, employing a modified Navon task (Navon, 1977) to compare global versus local processing in participants randomly assigned to wear formal or informal clothes . Their hypothesis was that enhanced abstract processing would selectively improve global perceptual processing. While they did find that those informal clothing performed better with global targets than local targets, these participants were non-significantly slower on global targets than those in casual clothing, and performed significantly worse with local targets, suggesting the clothing may hinder local processing rather then benefitting global. Additionally, a pair of preregistered studies intended to further explore this impact of formal clothing on abstract processing was unable to replicate the primary effect in two attempts using the original protocol, though they did find a 
relation under a variation in methodology (Burger \& Bless, 2017). The authors concluded that this effect may be more malleable and difficult to study than previously anticipated.

One of the most challenging aspects of conducting experiments on enclothed cognition is preventing hypothesis guessing from participants who are asked to wear specific articles of clothing. Kraus and Mendes (2014) randomly assigned male participants to wear either a business suit or sweatpants and a t-shirt during a negotiation task, and they provided cover for the manipulation by sewing bogus sensors into the clothing and explaining the task as a validation of the sensing technology. They found that those assigned to wear the sweatpants showed significantly depressed testosterone levels and made significantly more concessions than those in a business suit.

Regarding the potential effects of clothing on personality, Ellis and Jenkins (2015) found that wearing a watch is associated with higher levels of conscientiousness, arguing that watches can be considered a fashion accessory or expression of social status, thus falling under the umbrella of enclothed cognition. However, watch wearing was not randomly assigned nor manipulated in their study, so the authors were careful to note that a plausible explanation is simply that more conscientious people are more likely to wear a watch, strongly limiting the ability to provide support for the enclothed cognition hypothesis.

More convincing support comes from a pair of experiments where López-Pérez, Ambrona, Wilson, and Khalil (2016) provide evidence that wearing a nurse's tunic elevates empathic concern in comparison to either wearing the same tunic described as being that of a cleaner or merely being asked to identify with the nurse's tunic without wearing it. This work demonstrates the basic principle of enclothed cognition laid out by Adam and Galinsky: that proper enclothed effects depend on both the meaning attached to the clothing and on whether or not participants actually wear it. However, it is debatable as to whether this effect supports the claim that enclothed cognition influences not just what we think, but how we think. It is possible that empathic concern is more sensitive to this form of priming than is attentional control.

In one of the most recent investigations of these phenomena, Civile and Obhi (2017) had participants wear either a police or a mechanic's uniform while performing two different attentional tasks. Both tasks involved task irrelevant stimuli that were either an African-American or Caucasian face, or an upper-body image (with no face) of someone in a suit or a hoodie (which was meant to represent high and low socioeconomic status). Participants wearing police uniforms had their attention significantly more drawn to the hoodie image than that of the suit, and there was no difference for the other participants. Like López-Pérez et al. (2016), they also found no significant difference for participants who merely saw the uniform sitting on the desk next to their computer.
While the authors concluded that participants were demonstrating "uniform induced attentional biases" consistent with a belief that police disproportionately target those of lower socio-economic standing, they were surprised that they found no significant differences for attention to faces of different races, in contrast to previous research using the same task (Eberhardt, Goff, Purdie, \& Davies, 2004). It is unclear why participants would mimic one perceived bias but not the other.

These studies demonstrate a breadth of experimental paradigms that all seek to explore the bounds of the enclothed cognition phenomenon. Some studies, most notably those by López-Pérez et al. (2016) and Civile and Obhi (2017), offer supporting evidence of effects that depend on both the significance of a clothing item and on participants actually wearing the outfit rather then merely looking at it or identifying with it. However, there remains relatively little evidence that such effects can influence our core cognitive processes.

The effects shown in many of these subsequent studies may also be more susceptible to demand effects: participants asked to wear a nurse's outfit may reason that they are expected to respond in a more empathetic fashion, and those dressed as police officers may expect that they are supposed to look out for suspicious characters like those wearing hoodies. It is harder to rationalize why participants asked to wear doctor's coats should do better at ignoring color names: this seems more clearly a case of improved cognitive performance rather than a stereotypical sensitivity to particular types of stimuli or responses. No subsequent work demonstrating similar effects of clothing on Stroop task performance have been published, with the only known replication attempt being an underpowered student project with some methodological variations that make comparisons challenging (Womack, 2016).

\section{Need for Replication}

The replication of experimental findings is crucial, especially when the potential implications are controversial with respect to dominant views of current research. If the claims of enclothed cognition that were proposed by Adam and Galinsky (2012) are theoretically and empirically accepted, they pose a challenge to current theories of attentional control. It is unclear by what mechanism the significance of an item of clothing could improve a participant's capability to ignore or suppress the conflicting information coming from the meaning of the printed word in the Stroop task. In their discussion of experiments 2 and 3, the authors assert that these improvements in performance appear not to be caused by greater effort, but rather by an increase in the mental abilities themselves. This relatively easy improvement would appear to contrast sharply with the general failure of brain training games to produce performance gains on cognitive tasks relative to control conditions (Kable et al., 2017). 
There are also some aspects of Adam and Galinsky's results that merit further inspection. They found no interaction or main effect of lab coat on response time, the traditional measure of Stroop interference, and there was no main effect of lab coat on accuracy. Rather than finding that lab coats improved attention across the board, they found an interaction between stimulus type and lab coat: it was only for the incongruent trials (the more difficult ones) that participants with lab coats made fewer errors than those who did not wear a lab coat. In the congruent and neutral trials, those wearing a lab coat made a non-significantly larger number of errors.

Their methodology also differs from traditional Stroop design in several important ways. First of all, they used more incongruent trials than congruent trials, thus making the putatively irrelevant dimension of word probabilistically predictive of the response, font color. This has been shown to artificially inflate Stroop interference (Dishon-Berkovits \& Algom, 2000). Secondly, they collapsed across the congruent and neutral trials in all of their analyses, confounding the separate effects of Stroop interference (incongruent - neutral) and Stroop facilitation (neutral - congruent).

One potential explanation for the lack of a main effect is that accuracy was close to ceiling: participants typically only made a single incorrect response in the incongruent trials if they were wearing a lab coat and on average made two errors in the incongruent trials if they were not wearing a lab coat. More than half of the participants made no errors at all in either the neutral or congruent trials. This provides another motivation for utilizing response time as the primary dependent measure, as it does not have the same kind of ceiling that accuracy does on this task.

Adam and Galinsky's (2012) paper has attracted scientific interest (over 200 citations in six years since publication) and media attention, with the highest Altmetric attention score of any article from the Journal of Experimental Social Psychology published to date. Accordingly, further clarification about the size of such an effect is valuable. We reached out to the authors, who kindly provided us more information about the materials and procedures used in the original study and also provided their dataset for analysis. Their generosity allows us to directly replicate the experimental design of the original study and make direct statistical comparisons of their dataset to ours. We utilize more powerful statistical analyses extending beyond originally reported results to incorporate well established trial by trial effects, which can serve as a positive control to demonstrate statistical power of our data set. Our replication study was preregistered and all materials and data are available through the Open Science Foundation at osf.io/yw7d9

Because standard null hypothesis tests are incapable of supporting a claim of no effect, we make use of equivalence testing (Lakens, Scheel, \& Isager, 2018), which tests whether an effect is at least as extreme as the Smallest Effect
Size Of Interest (SESOI). As recommended for replication attempts by Simonsohn (2015), the SESOI here is computed as the effect size that the original design would have only had $33 \%$ power to detect (2:1 odds against). When the effect size found in the replication is significantly smaller than this threshold, the effect is deemed to be too small to have been meaningfully measured by the original study. Simonsohn calls this the small telescopes approach, and provides this allegory: presume a person claims to spot a new planet using their telescope. To evaluate their claim, get a bigger telescope and search the same space in the sky. If the larger telescope does not find a planet, this does not necessarily prove there is no planet there. What it can tell you is that if there is a planet there, the signal would have to be too faint to be reliably measured using the original smaller telescope. Thus, rather then attempting to prove that there is absolutely zero effect of wearing a lab coat, we instead will attempt to reject the null hypothesis of an effect at least as large as the above defined SESOI.

\section{Experiment}

This preregistered direct-replication attempt followed the replication recipe of Brandt et al. (2014), and was conducted across multiple labs, with independent data sets collected at four US Universities, with each receiving approval from their respective Institutional Review Board. All investigators used the same experimental software and procedures. All measures, manipulations, and exclusions in this study are reported here.

\section{Participants}

Participants at all four sites were undergraduates at their respective institutions. There were a total of 200 participants, with 66 participants from site 1, 61 from site 2, 46 from site 3 , and 27 from site 4 . Participants received credit in a psychology course as incentive to participate, but received no other compensation. After they were welcomed to the lab, they were provided with the informed consent form to sign. Participants were randomly assigned to the coat (100) or no coat (100) conditions. Participants in the coat condition were told the same cover story from Adam and Galinsky (2012); previous participants wore a lab coat to protect their clothes while the lab was under construction, and although the construction was now finished, they were asked to wear the coat for consistency. All participants were then seated at a computer to complete the experiment.

\section{Stimuli}

Following the original paper, this experiment used six stimuli. Congruent stimuli were the words "RED" and "BLUE" in their respective "ink" colors, incongruent stimuli were the same words in the opposite color, and neutral 
stimuli were the string "XXXX" in each of the two colors. The stimuli were displayed on monitors such that the vertical height of the letters subtended 2 degrees of visual angle based on participants' distance from the screen. Participants were tasked with identifying the ink color by pressing " $r$ " for red and "b" for blue.

\section{Procedure}

While the original paper used 20 incongruent trials, 15 congruent, and 15 neutral, this experiment balanced these and tripled the total number of trials by using 50 of each type. These 150 trials took about 10 minutes to complete. No feedback was provided during the experiment, but participants did receive feedback for an additional set of 12 practice trials at the beginning (which were excluded from analysis). There was a two-second interstimulus interval with a blank screen between every trial, followed by a one-second fixation cross, after which the stimulus appeared until a response was made. The experiment was programmed using the opensource PsychoPy experimental software (Peirce, 2007), and all of the related files can be downloaded through the Open Science Foundation at osf.io/yw7d9

\section{Results}

Unless otherwise noted, all analyses have been preregistered. Re-analysis of the original data was made possible through the generous cooperation of the authors. We report all measures, manipulations and exclusions for this study. The preregistration and all data and code used for data processing and analysis is available through the Open Science Foundation at osf.io/yw7d9.

\section{Data Cleaning and Descriptive Statistics}

The full data set (combined across locations) includes 200 participants with 150 trials each for a total of 30,000 trials. The preregistered sample size target was 58 per data collection site, but due to difficulties recruiting enough participants at several sites, we stopped at 200 without conducting preliminary analyses of the data. As decided in preregistration, trials with response times faster than 250 ms were excluded: there were only 29 of these, and their accuracy was $62 \%$. Although no upper cut off for slow responses was specified in the preregistration, this was deemed necessary, since the data included response times as long as 20,000 ms, yielding a skew of 11.94 and a kurtosis of 402.31 . It was decided to set a conservative boundary at three standard deviations above the mean for each participant. This was done rather than picking a single upper threshold in order to avoid selectively excluding trials from slower participants. There were 557 trials slower than this upper bound, accounting for less than $2 \%$ of all trials.

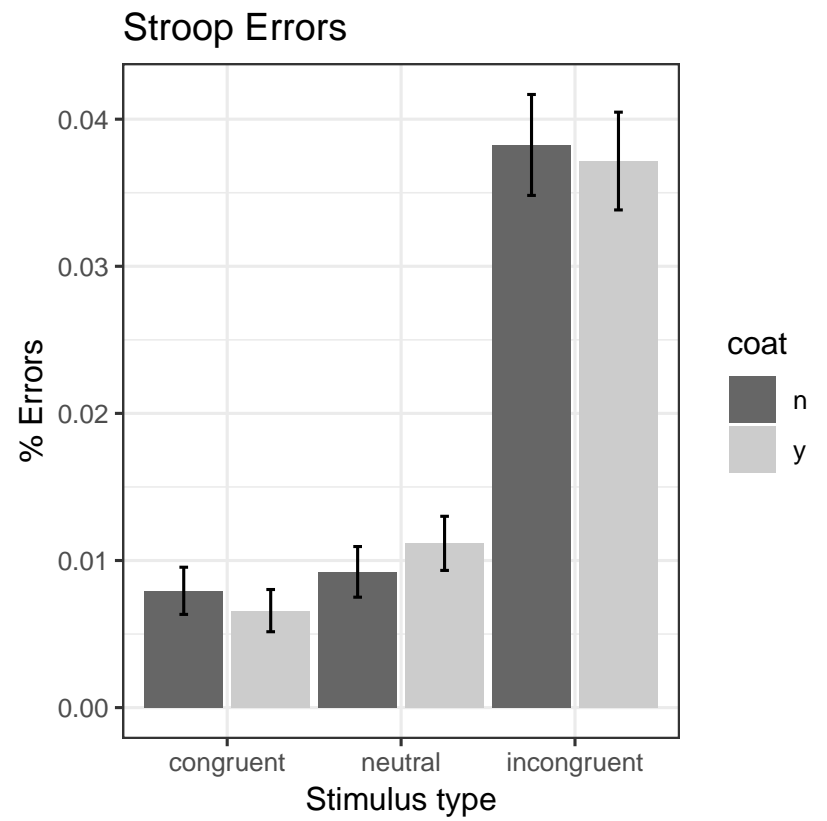

Figure 1. Percentage of incorrect responses as a function of stimulus condition and lab coat.

Additionally, exceptionally slow participants were excluded so as to not bias the mean. These were defined as those participants whose average response time was more than two standard deviations above the mean. There were seven of these participants, with an average reaction time of $1327.79 \mathrm{~ms}$ between them. Amongst the remaining participants, all except one had accuracy greater than $90 \%$, and the one with accuracy of only $79 \%$ was excluded as well, leaving 192 participants. In the remaining data set, which includes $94 \%$ of the total trials, the average accuracy was $98 \%$ and the response times for correct trials had $\mu=584.49$ $\mathrm{ms}, S D=246.24$, skew $=2.12$, and kurtosis $=8.00$. To ensure these particular exclusion criteria are not driving the specific results obtained, the critical tests below are also performed with the full data set for comparison.

\section{Stroop Analyses}

The effects of stimulus condition and lab coat on error rates are shown in Figure 1 For direct comparison with the analyses performed by Adam and Galinsky (2012), the neutral and congruent conditions were combined for replication analyses. A mixed-design ANOVA was conducted with lab coat as a between-participants factor and stimulus condition as a within-participants factor, with accuracy (averaged across trials) as the dependent measure. Stroop interference was evidenced by a main effect of stimulus condition with more errors for incongruent trials, $F(1,190)=$ $88.83, p<.001, \eta_{G}^{2}=.17$, but there was no significant main effect of lab coat, $F(1,190)=0.01, p=.91, \eta_{G}^{2}<.001$, 


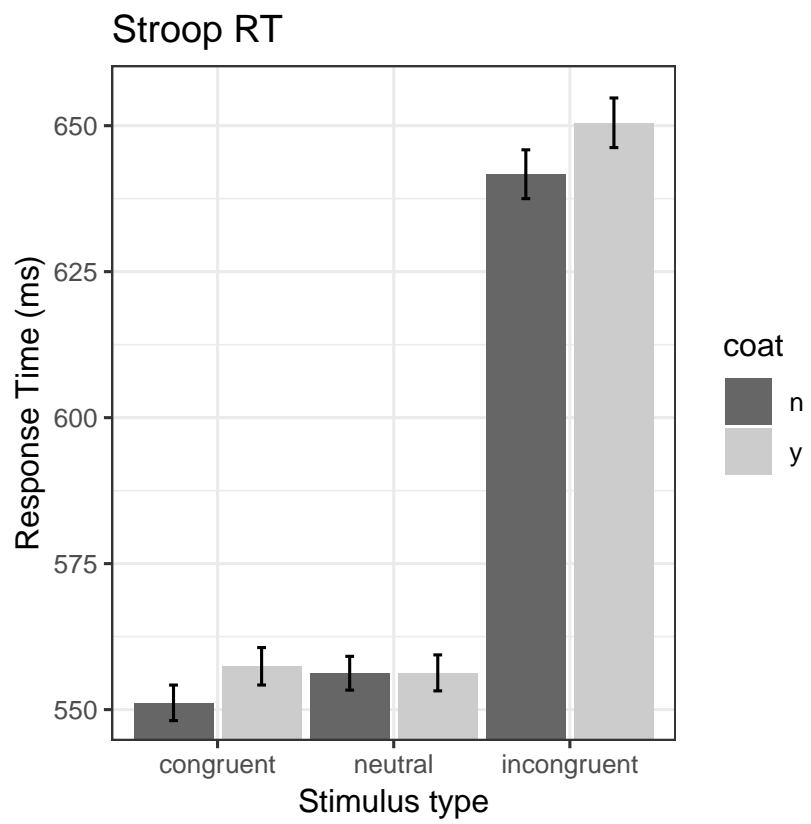

Figure 2. Average response time as a function of stimulus condition and lab coat.

nor an interaction, $F(1,190)=0.05, p=.83, \eta_{G}^{2}<.001$. Results were similar for the full data set (not using the exclusion criteria), with a main effect of stimulus condition, $F(1,198)=62.68, p<.001, \eta_{G}^{2}=.12$, no significant main effect of lab coat, $F(1,198)=0.22, p=.63, \eta_{G}^{2}<.001$, and no interaction, $F(1,198)=0.42, p=.52, \eta_{G}^{2}<.001$.

While Adam and Galinsky (2012) reported that response times "did not vary across conditions", they may have been referring to an absence of any effects of lab coat, as a mixed design ANOVA of their data shows a significant main effect of stimulus condition, $F(1,56)=43.41, p<.001, \eta_{G}^{2}=.076$. The absence of such an effect would have been puzzling, as this is the most common metric for quantifying Stroop interference (accuracy is much less commonly used as the dependent variable).

Our new data set shows similar results, seen in Figure 2 with a comparably sized effect of stimulus condition on RT, $F(1,190)=236.42, p<.001, \eta_{G}^{2}=.07$, and no significant effects of lab coat, $F(1,190)=0.07, p=.79, \eta_{G}^{2}<.001$ or interaction $F(1,190)=0.22, p=.64, \eta_{G}^{2}<.001$. Again, results were largely unchanged when using the full data set, with a main effect of stimulus condition, $F(1,198)=$ $231.61, p<.001, \eta_{G}^{2}=.06$, no main effect of lab coat, $F(1,198)=0.34, p=.56, \eta_{G}^{2}=.001$, and no interaction $F(1,198)=0.58, p=.45, \eta_{G}^{2}<.001$.

One limitation in comparing response times across these data sets is that since the original data has already been collapsed across trials, not only do we lose any characterization of within-participant variability, but we also lose the abil- ity to look at the response times for correct trials only, as is the standard practice. Fortunately, since error rates are low, this should have a minimal effect on the results, especially since we are looking at response time differences across conditions.

\section{Equivalence Test}

Because Adam and Galinsky only found a significant interaction between condition and lab coat for accuracy data, our replication analyses will not use response times. In order to conduct an equivalence test capable of rejecting the existence of effect sizes above a specified threshold, both the new and original data sets were fit with a generalized linear mixed-effect model on the trial-by-trial data, where the binary outcome variable was whether the response for a given trial was correct. Lab coat (yes or no) and stimulus condition (incongruent or neutral/congruent) were modeled as dichotomous fixed effects, with a random intercept for each participant and a by-participant random slope for stimulus condition.

Although Adam and Galinsky's original data were aggregated across trials, a trial-by-trial reconstruction was made by multiplying the percentage of errors in each condition by the number of trials in that condition. This approach enables the models to account for the reduction in within-participant variability afforded by obtaining more trials per participant, information which is lost in the standard ANOVA using averaged accuracy. Regardless, results from this approach largely matched the mixed-design ANOVA with accuracy averaged across trials.

In this new analysis of Adam and Galinsky's data, stimulus condition was a significant predictor, $z=3.93, p<.001$, with no significant effect of Lab coat, $z=1.08, p=.28$, but a significant interaction, $z=2.15, p=.03$. The odds ratios indicate that for those not wearing a coat, incongruent trials were 4.48 times more likely to result in error, but for those wearing lab coats incongruent trials were only 1.66 times more likely to result in a error. This same analysis for the new data set collected in this replication found a similar sized effect of stimulus condition, $z=7.52, p<.001$, with incongruent trials 4.42 times as likely to result in error. However, no significant effects were found for either lab coat $(z=.17, p=.86)$ or the interaction $(z=.07, p=.94)$. An analysis combining the new data with that of Adam and Galinsky also indicates an effect of stimulus condition, $z=8.76, p<.001$, with no significance for either lab coat $(z=.54, p=.59)$ or the interaction $(z=1.04, p=.30)$.

The Smallest Effect Size Of Interest (SESOI) for our equivalence test was determined according to the small telescopes argument (Simonsohn, 2015) as that which the original design had $33 \%$ power to detect. A power analysis using 10,000 simulations of the original design indicates that it would have had $33.8 \%$ power $(95 \%$ CI from $32.9 \%$ to $34.8 \%$ ) 


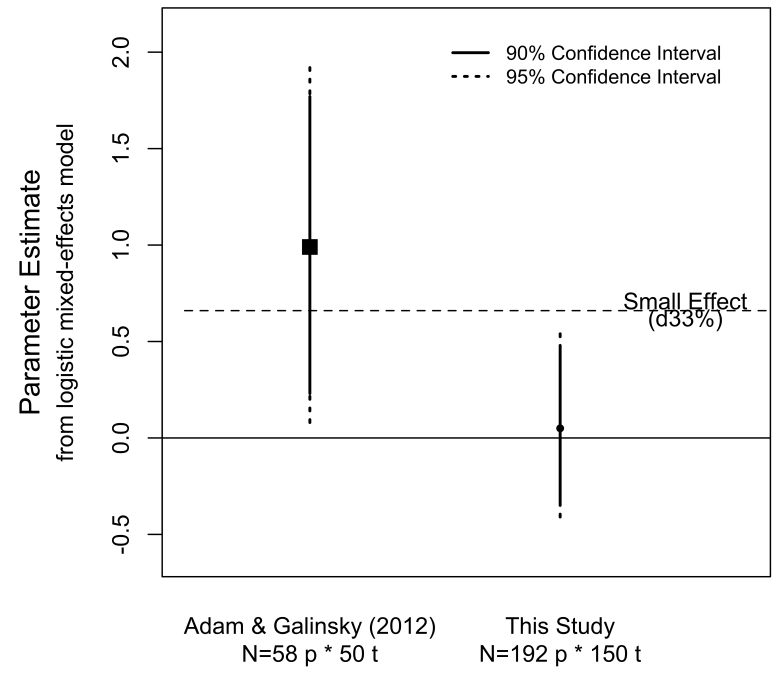

Figure 3. Comparison of interaction term parameter estimates for original study and replication. $\mathrm{N}$ is shown as number of participants multiplied by the number of trials.

to detect an interaction effect of .66, so this was taken as our SESOI. In order to conduct an equivalence test, we computed confidence intervals around the estimates of the interaction term in each model. Figure 3 shows these parameter estimates for both data sets. Because this is a logistic regression model, parameter estimates can be exponentiated to yield an odds ratio. The $90 \%$ CI for the original data set is $[.23,1.77]$, while in the new data set it is $[-.38, .41]$. Because the SESOI is outside of the $90 \%$ CI of the new estimate, we can conclude that the effect of stimulus condition is statistically equivalent for those wearing lab coats and those not (Lakens et al. 2018). More specifically, we can reject the null hypothesis that the interaction effect is at least as large as our Smallest Effect Size Of Interest.

One possible explanation for the difference between the replication data and the original experiment, as anticipated in the preregistration, is that the replication used 150 trials instead of the original 50. It is possible that the increased duration of the experiment decreased the effect of wearing the lab coat. In order to test for this possibility, an additional variable was added to the replication data dividing it into three blocks of 50 trials. The generalized linear model used previously was refit with block number as an additional predictor, but this model failed to explain significantly more variance than the simpler model, $\chi^{2}(1)=.62, p=.43$.

While the original experiment pooled data from neutral and congruent trials, some past examinations of the Stroop task have shown facilitatory effects of congruent trials when compared with neutral stimuli (MacLeod, 1991). In this replication experiment, separately fitting parameters for neutral trials and congruent trials increases the degrees of free- dom from 10 to 15 and still does not describe significantly more variance, $\chi^{2}(5)=8.40, p=.14$, so they were left pooled.

\section{Sequential Analyses}

To ensure that our experimental methodology could successful identify other documented effects in the Stroop paradigm, sequential effects on response time were analyzed as a positive control.

Because response times are well known to be non-normal, they were fit by a generalized mixed-effects model using an Inverse-Gaussian distribution with an identity link function, as recommended by Lo and Andrews (2015). The best fitting model had significant fixed effects $(p<.001$ for all) of condition, condition on the previous trial, and ink repeat, with an interaction between ink repeat and word repeat (which did not have a significant main effect) and an interaction between condition and previous condition. This model also included a random intercept for each participant with random by-participant slopes for condition.

As with accuracy, there was no further variance explained if block number was incorporated into the model, $\chi^{2}(1)=$ $.43, p=.51$, once again arguing against meaningful practice effects. The neutral and congruent conditions were left pooled, as there was no significant difference between them, $t(190)=.67, p=.51$.

\section{Discussion}

This preregistered direct-replication attempt casts doubt on the reported effect of enclothed cognition on selective attention. In our data set, with three times as many trials per participant and more than three times the number of participants as the original experiment (Adam \& Galinsky, 2012), wearing a lab coat had no significant effect on either response time or accuracy in the Stroop task, be it directly or through an interaction with stimulus condition. While it is logically impossible to demonstrate that the previously found interaction effect does not exist (is exactly equal to zero), equivalence testing indicates this effect is smaller than the Smallest Effect Size Of Interest (SESOI) as determined by the small telescopes argument (Simonsohn, 2015). Such a small effect could theoretically still be of interest to researchers, but is too small to have been reliably measured using Adam and Galinsky's original design.

We wish to be clear that we are not attempting to claim that clothing has no behavioral impacts on a participant. Previous research clearly indicates that different clothing choices can change the way that participants describe themselves (Hannover \& Kuhnen, 2002) and other measures of self reflection. Furthermore, studies such as those by LópezPérez et al. (2016) and Civile and Obhi (2017) indicate measurable effects that appear to depend on both the significance attached to the clothing and that participants actually wear 
it. However, we wish to call into question the stronger claim that enclothed cognition has demonstrated effects on how we think rather than just what we think. Although the subsequent studies have demonstrated how clothing may bias attention toward certain types of stimuli or alter the tendency to display helping behaviors, these effects may be at least somewhat elicited by demand effects and seem meaningfully different from Adam and Galinsky's claim of a fundamental improvement in selective attention.

If this effect is small enough to have less than a one in three chance of being detected under the original design, one may ask how a statistically significant result was found. False alarms are of course always a possibility, and while it is difficult to determine the factors that may contribute to them in any particular case, one possible factor at play here is the number of tests that could have been said to show significant results in favor of the hypothesis. While the authors asserted that an interaction between lab coat and trial type was what they had expected based on their theory of enclothed cognition, it seems reasonable that finding a main effect of lab coat instead could have also been taken as support for the theory. Additionally, having two potential dependent variables of response time and accuracy means that there are four separate tests that could have plausibly been claimed to confirm the theory, thus raising the chance of a false alarm overall to around $18.5 \%$ (subject to dependencies between tests). This important problem can be ameliorated by either preregistering the intended tests that researchers plan to use to declare the success of a manipulation (and correcting for multiple comparisons), and/or conducting a confirmatory experiment after an initial exploratory phase.

A potential alternative explanation for the difference is that the effect of wearing a lab coat has changed over time since the original study. Although the participant populations were similar demographically between this experiment and the original (undergraduate students, most of whom were enrolled in a psychology course), an argument could be made that publicity surrounding the so-called replication crisis has reduced the perceived associations between lab coats and intelligence. There have also been growing trends of antiintellectualism in the American political climate which could contribute to a change. While this seems unlikely to fully account for the differences in results, the possibility of such a history effect should be noted.

The sequential effects on response time seen here are as expected based on the work by Huettel and Lockhead (1999) and more recently Little, Wang, and Nosofsky (2016) using a related selective attention task, the Garner paradigm. Their work predicts our finding that trials where both font color and word were repeated are faster than all other trials (by $18 \mathrm{~ms}$ ), while trials in which the stimulus changes but the response stays the same (font color repeat with word change) are the slowest of all. Our data also shows the commonly found
Congruence Sequence Effect (sometimes called the Gratton effect), where Stroop interference is diminished following incongruent trials (Duthoo, Abrahamse, Braem, Boehler, \& Notebaert 2014). This effect is often understood in terms of conflict-monitoring theory, with greater cognitive control elicited by the conflict inherent in incongruent stimuli.

The detection of these small but significant sequential effects as expected from the literature helps to advance the argument that lack of power or poor experimental methodology are not the reasons why we did not detect an effect of wearing a lab coat. Proponents of enclothed cognition now have the task of demonstrating domains we may still expect to find its effects, and establishing a theoretical basis for why those domains may be impacted while others, like selective attention, are not.

\section{Acknowledgments}

The authors wish to thank Jane Hwang, Olivia Pettengill, Kylie Schiloski, Shelby Brown, Jason Smith, and the students of Dr. Burns' Research Methods class for help with data collection.

\section{Open Practices}

The experiment in this article earned Open Materials, Open Data, and Preregistration badges for transparent practices. Materials, data, and preregistered plan for the experiment are available at osf.io/yw7d9

\section{References}

Adam, H., \& Galinsky, A. D. (2012). Enclothed cognition. Journal of Experimental Social Psychology, 48(4), 918-925.

Barsalou, L. W. (2010). Grounded cognition: Past, present, and future. Topics in Cognitive Science, 2(4), 716724.

Brandt, M. J., IJzerman, H., Dijksterhuis, A., Farach, F. J., Geller, J., Giner-Sorolla, R., . . . van 't Veer, A. (2014). The replication recipe: What makes for a convincing replication? Journal of Experimental Social Psychology, 50, 217-224.

Burger, A. M., \& Bless, H. (2017, August). Cognitive consequences of formal clothing: the effects of clothing versus thinking of clothing. Comprehensive Results in Social Psychology, 2(2-3), 228-252.

Civile, C., \& Obhi, S. S. (2017, February). Students wearing police uniforms exhibit biased attention toward individuals wearing hoodies. Frontiers in Psychology, 8, $1-14$.

DeCaro, M. S., Van Stockum, C. A., \& Wieth, M. B. (2016). When higher working memory capacity hinders insight. Journal of experimental psychology. Learning, memory, and cognition, 42(1), 39-49. 
Dishon-Berkovits, M., \& Algom, D. (2000). The Stroop effect: It is not the robust phenomenon that you have thought it to be. Memory and Cognition, 28(8), 14371449.

Duthoo, W., Abrahamse, E. L., Braem, S., Boehler, C. N., \& Notebaert, W. (2014). The heterogeneous world of congruency sequence effects: an update. Frontiers in Psychology, 5, 1-9.

Eberhardt, J. L., Goff, P. A., Purdie, V. J., \& Davies, P. G. (2004). Seeing black: Race, crime, and visual processing. Journal of Personality and Social Psychology, 87(6), 876-893.

Ellis, D. A., \& Jenkins, R. (2015). Watch-wearing as a marker of conscientiousness. PeerJ, 3(3), e1210-11.

Hannover, B., \& Kuhnen, U. (2002). "The clothing makes the self" via knowledge activation. Journal of Applied Social Psychology, 32(12), 2513-2525.

Huettel, S. A., \& Lockhead, G. R. (1999). Range effects of an irrelevant dimension on classification. Perception and Psychophysics, 61(8), 1624-1645.

Hur, Y., Sturdee, M., Alonso, M. B., Markopoulos, P., \& Alexander, J. (2017). Fiction and physicality: A designerly approach towards complexities of emerging technologies. The Design Journal, S3849-S3862.

Kable, J. W., Caulfield, M. K., Falcone, M., McConnell, M., Bernardo, L., Parthasarathi, T., ... Lerman, C. (2017). No effect of commercial cognitive training on brain activity, choice behavior, or cognitive performance. Journal of Neuroscience, 37(31), 7390-7402.

Kraus, M. W., \& Mendes, W. B. (2014). Sartorial symbols of social class elicit class-consistent behavioral and physiological responses: A dyadic approach. Journal of Experimental Psychology: General, 143(6), 23302340.

Lakens, D., Scheel, A. M., \& Isager, P. M. (2018). Equivalence Testing for Psychological Research: A Tutorial. Advances in Methods and Practices in Psychological Science, 1(2), 259-269.

Lee, S. W. S., \& Schwarz, N. (2012). Bidirectionality, mediation, and moderation of metaphorical effects: The embodiment of social suspicion and fishy smells. Journal of Personality and Social Psychology, 103(5), 737-749.

Little, D. R., Wang, T., \& Nosofsky, R. M. (2016). Sequence-sensitive exemplar and decision-bound accounts of speeded-classification performance in a modified Garner-tasks paradigm. Cognitive Psychology, 89, 1-38.

Lo, S., \& Andrews, S. (2015). To transform or not to trans- form: using generalized linear mixed models to analyse reaction time data. Frontiers in Psychology, 6, 116.

López-Pérez, B., Ambrona, T., Wilson, E. L., \& Khalil, M. (2016). The effect of enclothed cognition on empathic responses and helping behavior. Social Psychology, 47(4), 223-231.

MacLeod, C. M. (1991). Half a century of research on the Stroop effect: an integrative review. Psychological Bulletin, 109(2), 163-203.

MacLeod, C. M. (1992). The Stroop task: The "gold standard" of attentional measures. Journal of Experimental Psychology: General, 121(1), 12-14.

Morris, T. L., Gorham, J., Cohen, S. H., \& Huffman, D. (1996). Fashion in the classroom: Effects of attire on student perceptions of instructors in college classes. Communication Education, 45(2), 135-148.

Navon, D. (1977). Forest before trees: The precedence of global features in visual perception. Cognitive Psychology, 9(3), 353-383.

Pagliarini, L., \& Lund, H. H. (2011). Wearable playware. In 2011 8th international conference on ubiquitous robots and ambient intelligence (urai) (pp. 9-13). IEEE.

Peirce, J. W. (2007). PsychoPy-psychophysics software in Python. Journal of Neuroscience Methods, 162, 8-13.

Pomplun, M., Reingold, E. M., \& Shen, J. (2001). Investigating the visual span in comparative search: the effects of task difficulty and divided attention. Cognition, 81(2), B57-B67.

Simonsohn, U. (2015). Small telescopes: Detectability and the evaluation of replication results. Psychological Science, 26(5), 559-569.

Slepian, M. L., Ferber, S. N., Gold, J. M., \& Rutchick, A. M. (2015, February). The cognitive consequences of formal clothing. Social Psychological and Personality Science, 6(6), 661-668.

Stroop, J. R. (1935). Studies of interference in serial verbal reactions. Journal of Experimental Psychology, 18(6), 643-662.

Van Stockum, C. A., \& DeCaro, M. S. (2014). Enclothed cognition and controlled attention during insight problem-solving. The Journal of Problem Solving, 7(1), 73-83.

Womack, R. (2016). Enclothed cognition: The effect of attire on attention task performance. Samford Undergraduate Research Journal, 94-103. 